\title{
Dynamics of an Inverting Tippe Top
}

Stefan RAUCH-WOJCIECHOWSKI and Nils RUTSTAM

Department of Mathematics, Linköping University, Linköping, Sweden

E-mail: strau@mai.liu.se, ergoroff@hotmail.com

Received September 05, 2013, in final form February 18, 2014; Published online February 27, 2014

http://dx.doi.org/10.3842/SIGMA.2014.017

\begin{abstract}
The existing results about inversion of a tippe top (TT) establish stability of asymptotic solutions and prove inversion by using the LaSalle theorem. Dynamical behaviour of inverting solutions has only been explored numerically and with the use of certain perturbation techniques. The aim of this paper is to provide analytical arguments showing oscillatory behaviour of TT through the use of the main equation for the TT. The main equation describes time evolution of the inclination angle $\theta(t)$ within an effective potential $V(\cos \theta, D(t), \lambda)$ that is deforming during the inversion. We prove here that $V(\cos \theta, D(t), \lambda)$ has only one minimum which (if Jellett's integral is above a threshold value $\lambda>\lambda_{\text {thres }}=\frac{\sqrt{m g R^{3} I_{3} \alpha}(1+\alpha)^{2}}{\sqrt{1+\alpha-\gamma}}$ and $1-\alpha^{2}<\gamma=\frac{I_{1}}{I_{3}}<1$ holds) moves during the inversion from a neighbourhood of $\theta=0$ to a neighbourhood of $\theta=\pi$. This allows us to conclude that $\theta(t)$ is an oscillatory function. Estimates for a maximal value of the oscillation period of $\theta(t)$ are given.
\end{abstract}

Key words: tippe top; rigid body; nonholonomic mechanics; integrals of motion; gliding friction

2010 Mathematics Subject Classification: 70F40; 74M10; 70E18; 70E40; 37B25

\section{Introduction}

A tippe top (TT) is constructed as a truncated axisymmetric sphere with a small peg as its handle. The top is spun on a flat surface with the peg pointing upward. If the initial rotation is fast enough, the top will start to turn upside down until it ends up spinning on its peg. We call this interesting phenomenon an inversion.

It is known that the TT inverts when the physical parameters satisfy the conditions $1-\alpha<$ $\gamma=\frac{I_{1}}{I_{3}}<1+\alpha$ where $0<\alpha<1$ is the eccentricity of the center of mass and $I_{1}, I_{3}$ are the main moments of inertia.

The TT and the inversion phenomenon has been studied extensively throughout the years, but the dynamics of inversion has proven to be a difficult problem. This is because even the most simplified model for the rolling and gliding TT is a non-integrable dynamical system with at least 6 degrees of freedom. The focus in many works has been on the asymptotics of the TT $[1,5,9,10,13]$ or on numerical simulations for a TT $[3,11,18]$.

In this paper we study equations of motion for a rolling and gliding TT in the case of inverting solutions and analyse dynamical properties of such solutions through the main equations for the TT $[12,14]$.

We study the main equation for the TT for a subset of parameters satisfying $1-\alpha^{2}<$ $\gamma<1$ and $\frac{1-\gamma}{\gamma+\alpha^{2}-1}=\frac{m R^{2}}{I_{3}}$ when it acquires a simpler form, which enables detailed analysis of deformation of the effective potential $V(\cos \theta, D, \lambda)$ during the inversion. We show that, during the inversion, a minimum of the effective potential moves from the neighbourhood of $\theta=0$ to the neighbourhood of $\theta=\pi$ and therefore the inclination angle $\theta(t)$ oscillates within a nutational band that moves from the north pole to the south pole of the unit sphere $S^{2}$. We give also estimates for the period of nutation of the symmetry axis. 


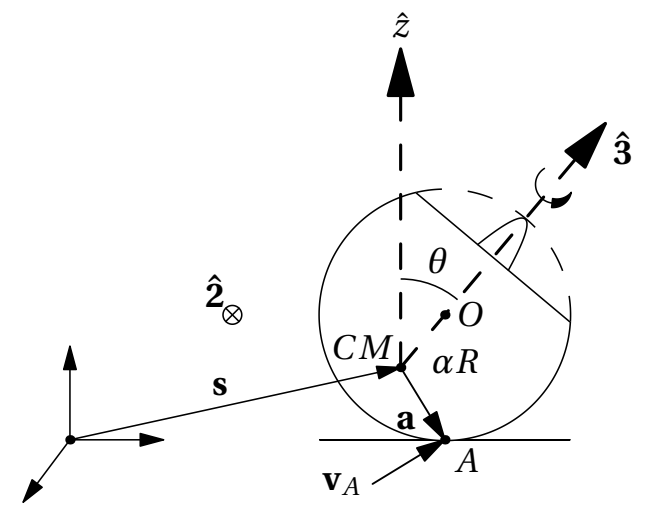

Figure 1. Diagram of the TT. Note that $\mathbf{a}=R \alpha \hat{\mathbf{3}}-R \hat{z}$.

\section{The tippe top model}

We model the TT as an axisymmetric sphere of mass $m$ and radius $R$ which is in instantaneous contact with the supporting plane at the point $A$. The center of mass $C M$ is shifted from the geometric center $O$ along its symmetry axis by $\alpha R$, where $0<\alpha<1$.

We choose a fixed inertial reference frame $(\widehat{X}, \widehat{Y}, \widehat{Z})$ with $\widehat{X}$ and $\widehat{Y}$ parallel to the supporting plane and with vertical $\widehat{Z}$. We place the origin of this system in the supporting plane. Let $(\hat{x}, \hat{y}, \hat{z})$ be a frame defined through rotation around $\widehat{Z}$ by an angle $\varphi$, where $\varphi$ is the angle between the plane spanned by $\widehat{X}$ and $\widehat{Z}$ and the plane spanned by the points $C M, O$ and $A$.

The third reference frame $(\hat{\mathbf{1}}, \hat{\mathbf{2}}, \hat{\mathbf{3}})$, with origin at $C M$, is defined by rotating $(\hat{x}, \hat{y}, \hat{z})$ by an angle $\theta$ around $\hat{y}$. Thus $\hat{\mathbf{3}}$ will be parallel to the symmetry axis, and $\theta$ will be the angle between $\hat{z}$ and $\hat{\mathbf{3}}$. This frame is not fully fixed in the body. The axis $\hat{\mathbf{2}}$ points behind the plane of the picture of Fig. 1.

We let $\mathbf{s}$ denote the position of $C M$ w.r.t. the origin of the frame $(\widehat{X}, \widehat{Y}, \widehat{Z})$ and the vector from $C M$ to $A$ is $\mathbf{a}=R(\alpha \hat{\mathbf{3}}-\hat{z})$. The orientation of the body w.r.t. the inertial reference frame $(\hat{X}, \hat{Y}, \hat{Z})$ is described by the Euler angles $(\theta, \varphi, \psi)$, where $\psi$ is the rotation angle of the sphere about the symmetry axis. With this notation, the angular velocity of the TT is $\boldsymbol{\omega}=-\dot{\varphi} \sin \theta \hat{\mathbf{1}}+\dot{\theta} \hat{\mathbf{2}}+(\dot{\psi}+\dot{\varphi} \cos \theta) \hat{\mathbf{3}}$, and we denote $\omega_{3}:=\dot{\psi}+\dot{\varphi} \cos \theta$.

The principal moments of inertia along the axes $(\hat{\mathbf{1}}, \hat{\mathbf{2}}, \hat{\mathbf{3}})$ are denoted by $I_{1}=I_{2}$ and $I_{3}$, so the inertia tensor $\mathbb{I}$ will have components $\left(I_{1}, I_{1}, I_{3}\right)$ with respect to the $(\hat{\mathbf{1}}, \hat{\mathbf{2}}, \hat{\mathbf{3}})$-frame. The axes $\hat{\mathbf{1}}$ and $\hat{\mathbf{2}}$ are principal axes due to the axisymmetry of TT. The equations of motion for TT are the Newton equations for the rolling and gliding rigid body

$$
m \ddot{\mathbf{s}}=\mathbf{F}-m g \hat{z}, \quad \dot{\mathbf{L}}=\mathbf{a} \times \mathbf{F}, \quad \dot{\hat{\mathbf{3}}}=\boldsymbol{\omega} \times \hat{\mathbf{3}},
$$

where $\mathbf{F}$ is the external force acting on the TT at the supporting point $A$ and $\mathbf{L}=\mathbb{I} \boldsymbol{\omega}$ is the angular momentum w.r.t. $C M$. We assume that the TT is always in contact with the plane at $A$, so $\hat{z} \cdot(\mathbf{a}+\mathbf{s})=0$ holds at all times. This system is known to admit Jellett's integral of motion $\lambda=-\mathbf{L} \cdot \mathbf{a}=R I_{1} \dot{\varphi} \sin ^{2} \theta-R I_{3} \omega_{3}(\alpha-\cos \theta$ ) (without loss of generality, we will assume in this paper that $\lambda$ is positive).

The contact condition determines the vertical part of the external force, but the planar parts must be specified to make system (1) complete. We assume that the contact force has the form $\mathbf{F}=g_{n} \hat{z}-\mu g_{n} \mathbf{v}_{A}$, where $g_{n} \geq 0$ is the normal force and $-\mu g_{n} \mathbf{v}_{A}$ is a viscous-type friction force, acting against the gliding velocity $\mathbf{v}_{A}$. The quantity $\mu(\mathbf{L}, \hat{\mathbf{3}}, \dot{\mathbf{s}}, \mathbf{s}, t) \geq 0$ is a friction coefficient.

For this model of the rolling and gliding TT, it is easy to see $[4,15]$ that the energy is decreasing $\dot{E}=\mathbf{F} \cdot \mathbf{v}_{A}<0$ and that the $\hat{y}$ component of the friction force is the only force creating the torque necessary for transferring the rotational energy into the potential energy, 
thus lifting the $C M$ of the TT. This mechanism shows that the inversion phenomenon is created by the gliding friction.

The asymptotic properties of this model have been analysed in previous works $[1,5,9,10,13]$. In the nongliding case, $\mathbf{v}_{A}=0$, the possible motions for the TT are either spinning in the upright $(\theta=0)$ or in the inverted $(\theta=\pi)$ position, or rolling around with fixed $C M$ with an inclination angle $\theta \in(0, \pi)$.

The inclined rolling solutions are called tumbling solutions. If $1-\alpha<\gamma<1+\alpha$, where $\gamma=I_{1} / I_{3}$, every angle in the interval $(0, \pi)$ determines an admissible tumbling solution. Further, by a LaSalle-type theorem [13], it is known that for initial conditions such that the absolute value of the Jellett integral $|\lambda|$ is above the threshold value $\lambda_{\text {thres }}$, only the inverted spinning position is a stable asymptotic solution. For a TT built such that it satisfies the parameter condition $1-\alpha<\gamma<1+\alpha$ and for initial conditions with $\mathbf{L} \cdot \hat{z}$ such that $\lambda>\lambda_{\text {thres }}$, the inversion can take place.

Since we are primarily interested in the dynamics of inversion and we want to consider solutions describing an inverting TT, the basic assumptions are that the TT in question satisfies the parameter constraint and that we have initial conditions such that $\lambda$ is above the threshold. Then we have a situation where an inverting solution becomes the only stable asymptotic solution, so the TT has to invert. Our aim is to describe the dynamics of inverting solutions.

In our particular model, the assumptions about the reaction force $\mathbf{F}$ and the contact constraint yield the reduced equations of motion for the rolling and gliding TT:

$$
\frac{d}{d t}(\mathbb{I} \boldsymbol{\omega})=\mathbf{a} \times\left(g_{n} \hat{z}-\mu g_{n} \mathbf{v}_{A}\right), \quad m \ddot{\mathbf{r}}=-\mu g_{n} \mathbf{v}_{A}, \quad \dot{\hat{\mathbf{3}}}=\boldsymbol{\omega} \times \hat{\mathbf{3}},
$$

where $\mathbf{r}=\mathbf{s}-s_{\hat{z}} \hat{z}$. We write the gliding velocity as $\mathbf{v}_{A}=\nu_{x} \cos \theta \hat{\mathbf{1}}+\nu_{y} \hat{\mathbf{2}}+\nu_{x} \sin \theta \hat{\mathbf{3}}$, where $\nu_{x}, \nu_{y}$ are the velocities in the $\hat{\mathbf{2}} \times \hat{z}$ and $\hat{\mathbf{2}}$ direction. Equations (2) can be written in the Euler form and then solved for the highest derivative of each of the variables $\left(\theta, \varphi, \omega_{3}, \nu_{x}, \nu_{y}\right)$. We then get the system

$$
\begin{aligned}
\ddot{\theta}= & \frac{\sin \theta}{I_{1}}\left(I_{1} \dot{\varphi}^{2} \cos \theta-I_{3} \omega_{3} \dot{\varphi}-R \alpha g_{n}\right)+\frac{R \mu g_{n} \nu_{x}}{I_{1}}(1-\alpha \cos \theta), \\
\ddot{\varphi}= & \frac{I_{3} \dot{\theta} \omega_{3}-2 I_{1} \dot{\theta} \dot{\varphi} \cos \theta-\mu g_{n} \nu_{y} R(\alpha-\cos \theta)}{I_{1} \sin \theta}, \\
\dot{\omega}_{3}= & -\frac{\mu g_{n} \nu_{y} R \sin \theta}{I_{3}}, \\
\dot{\nu}_{x}= & \frac{R \sin \theta}{I_{1}}\left(\dot{\varphi} \omega_{3}\left(I_{3}(1-\alpha \cos \theta)-I_{1}\right)+g_{n} R \alpha(1-\alpha \cos \theta)-I_{1} \alpha\left(\dot{\theta}^{2}+\dot{\varphi}^{2} \sin ^{2} \theta\right)\right) \\
& -\frac{\mu g_{n} \nu_{x}}{m I_{1}}\left(I_{1}+m R^{2}(1-\alpha \cos \theta)^{2}\right)+\dot{\varphi} \nu_{y}, \\
\dot{\nu}_{y}= & -\frac{\mu g_{n} \nu_{y}}{m I_{1} I_{3}}\left(I_{1} I_{3}+m R^{2} I_{3}(\alpha-\cos \theta)^{2}+m R^{2} I_{1} \sin ^{2} \theta\right) \\
& +\frac{\omega_{3} \dot{\theta} R}{I_{1}}\left(I_{3}(\alpha-\cos \theta)+I_{1} \cos \theta\right)-\dot{\varphi} \nu_{x},
\end{aligned}
$$

which, if we add the equation $\frac{d}{d t}(\theta)=\dot{\theta}$, becomes a dynamical system of the form

$$
\left(\dot{\theta}, \ddot{\theta}, \ddot{\varphi}, \dot{\omega}_{3}, \dot{\nu}_{x}, \dot{\nu}_{y}\right)=\left(h_{1}\left(\theta, \ldots, \nu_{y}\right), \ldots, h_{6}\left(\theta, \ldots, \nu_{y}\right)\right) .
$$

The value of the normal force $g_{n}$ can be determined from the contact constraint $(\mathbf{a}+\mathbf{s}) \cdot \hat{z}=0$ and it is

$$
g_{n}=\frac{m g I_{1}+m R \alpha\left(\cos \theta\left(I_{1} \dot{\varphi}^{2} \sin ^{2} \theta+I_{1} \dot{\theta}^{2}\right)-I_{3} \dot{\varphi} \omega_{3} \sin ^{2} \theta\right)}{I_{1}+m R^{2} \alpha^{2} \sin ^{2} \theta-m R^{2} \alpha \sin \theta(1-\alpha \cos \theta) \mu \nu_{x}} .
$$

We see that we get a complicated, nonlinear system for 6 unknowns. 


\section{The main equation for the tippe top}

For further study of inverting solutions we need to clarify the logic of applying the main equation for the tippe top (METT) to analysing motion of TT. We need also to recall properties of TT equations when the TT is only rolling on the supporting surface and the gliding velocity vanishes $\mathbf{v}_{A}=\dot{\mathbf{s}}+\boldsymbol{\omega} \times \mathbf{a}=0$. It is the well known [2, 7] integrable case of the rolling axisymmetric sphere that was first separated by Chaplygin. We need to explain how the structure of separation equations motivates the introduction of the METT and how this equation differs from the classical separation equation.

For the purely rolling axisymmetric sphere, the constraint $\mathbf{v}_{A}=\dot{\mathbf{s}}+\boldsymbol{\omega} \times \mathbf{a}=0$ implies that the equations of motion (1) reduce to a closed system for the vectors $\hat{\mathbf{3}}$ and $\boldsymbol{\omega}$ :

$$
\frac{d}{d t}(\mathbb{I} \boldsymbol{\omega})=m \mathbf{a} \times\left(g \hat{z}-\frac{d}{d t}(\boldsymbol{\omega} \times \mathbf{a})\right), \quad \dot{\hat{\mathbf{3}}}=\boldsymbol{\omega} \times \hat{\mathbf{3}} .
$$

For this system the external force is dynamically determined: $\mathbf{F}=m g \hat{z}-m \frac{d}{d t}(\boldsymbol{\omega} \times \mathbf{a})$. In the Euler angle form the equations give a fourth order dynamical system for $\left(\theta, \dot{\theta}, \dot{\varphi}, \omega_{3}\right)$.

The system (9) admits three integrals of motion. Since the system is conservative, the energy

$$
\begin{aligned}
E= & \frac{1}{2} m \dot{\mathbf{s}}^{2}+\frac{1}{2} \boldsymbol{\omega} \cdot \mathbf{L}+m g \mathbf{s} \cdot \hat{z}=\frac{1}{2}\left(I_{1} \dot{\varphi}^{2} \sin ^{2} \theta+I_{1} \dot{\theta}^{2}+I_{3} \omega_{3}^{2}\right)+m g R(1-\alpha \cos \theta) \\
& +\frac{1}{2} m R^{2}\left[(\alpha-\cos \theta)^{2}\left(\dot{\theta}^{2}+\dot{\varphi}^{2} \sin ^{2} \theta\right)+\sin ^{2} \theta\left(\dot{\theta}^{2}+\omega_{3}^{2}+2 \omega_{3} \dot{\varphi}(\alpha-\cos \theta)\right)\right]
\end{aligned}
$$

is an integral of motion. We also have Jellett's integral $\lambda=R I_{1} \dot{\varphi} \sin ^{2} \theta-R I_{3} \omega_{3}(\alpha-\cos \theta)$ as well as the Routh integral

$$
D:=\omega_{3} \sqrt{I_{3} I_{1}+m R^{2}(\alpha-\cos \theta)^{2}+m R^{2} I_{1} \sin ^{2} \theta}=I_{3} \omega_{3} \sqrt{d(\cos \theta)},
$$

where

$$
d(z)=\gamma+\sigma(\alpha-z)^{2}+\sigma \gamma\left(1-z^{2}\right), \quad \sigma=\frac{m R^{2}}{I_{3}} \quad \text { and } \quad \gamma=\frac{I_{1}}{I_{3}} .
$$

They allow to eliminate $\omega_{3}=\frac{D}{I_{3} \sqrt{d(\cos \theta)}}$ and $\dot{\varphi}=\frac{\lambda \sqrt{d(\cos \theta)}+R D(\alpha-\cos \theta)}{R I_{1} \sin ^{2} \theta \sqrt{d(\cos \theta)}}$ from the expression of the energy (10) to get the separation equation

$$
E=g(\cos \theta) \dot{\theta}^{2}+V(\cos \theta, D, \lambda)
$$

where $g(\cos \theta)=\frac{1}{2} I_{3}\left(\sigma\left((\alpha-\cos \theta)^{2}+1-\cos ^{2} \theta\right)+\gamma\right)$ and

$$
V(z=\cos \theta, D, \lambda)=m g R(1-\alpha z)+\frac{(\lambda \sqrt{d(z)}+R D(\alpha-z))^{2}}{2 I_{3} R^{2} \gamma^{2}\left(1-z^{2}\right)}+\frac{\left(R^{2} D^{2}-\sigma \lambda^{2}\right)}{2 R^{2} I_{1}} .
$$

The separable first order differential equation (11) for $\theta$ determines the motion of the rolling TT. It is the Chaplygin separation equation for an axisymmetric sphere [2]. We shall show that for certain choice of parameters the effective potential $V(z, D, \lambda)$ is convex in $z \in[-1,1]$ so, since $V(z, D, \lambda) \rightarrow \infty$ as $z \rightarrow \pm 1$, it has one minimum in the interval [ $-1,1]$. This means that for fixed $E$ the solutions $\theta(t)$ describe nutational motion of the rolling TT between two bounding angles $\theta_{1}, \theta_{2}$ determined by the equation $E=V(\cos \theta, D, \lambda)$. 
The rolling and gliding TT only has $\lambda$ as an integral of motion. It is useful however to consider $D\left(\theta(t), \omega_{3}(t)\right)=I_{3} \omega_{3}(t) \sqrt{d(\cos \theta(t))}$ being now a time dependent function. Its derivative we calculate using the equations of motion (3)-(7) for the rolling and gliding TT:

$$
\frac{d}{d t} D\left(\theta, \omega_{3}\right)=\frac{\gamma m R \sin \theta}{\sqrt{d(\cos \theta)}}\left(\dot{\varphi} \nu_{x}+\dot{\nu}_{y}\right)=\frac{\gamma m}{\alpha \sqrt{d(\hat{z} \cdot \hat{\mathbf{3}})}}(\hat{z} \times \mathbf{a}) \cdot \dot{\mathbf{v}}_{A} .
$$

For the total energy of TT, $E=\frac{1}{2} m\left(\mathbf{v}_{A}-\boldsymbol{\omega} \times \mathbf{a}\right)^{2}+\frac{1}{2} \boldsymbol{\omega} \cdot \mathbf{L}+m g \mathbf{s} \cdot \hat{z}$, we know that $\dot{E}=\mathbf{F} \cdot \mathbf{v}_{A}<0$. The part of $E$ that does not depend on $\mathbf{v}_{A}$,

$$
\begin{aligned}
\tilde{E}\left(\theta, \dot{\theta}, \dot{\varphi}, \omega_{3}\right)= & \frac{1}{2} m(\boldsymbol{\omega} \times \mathbf{a})^{2}+\frac{1}{2} \boldsymbol{\omega} \cdot \mathbf{L}+m g \mathbf{s} \cdot \hat{z} \\
= & \frac{1}{2}\left(I_{1} \dot{\varphi}^{2} \sin ^{2} \theta+I_{1} \dot{\theta}^{2}+I_{3} \omega_{3}^{2}\right)+m g R(1-\alpha \cos \theta) \\
& +\frac{1}{2} m R^{2}\left[(\alpha-\cos \theta)^{2}\left(\dot{\theta}^{2}+\dot{\varphi}^{2} \sin ^{2} \theta\right)+\sin ^{2} \theta\left(\dot{\theta}^{2}+\omega_{3}^{2}+2 \omega_{3} \dot{\varphi}(\alpha-\cos \theta)\right)\right]
\end{aligned}
$$

we will call the modified energy function. The derivative of this function is $\frac{d}{d t} \tilde{E}\left(\theta, \dot{\theta}, \dot{\varphi}, \omega_{3}\right)=$ $m \dot{\mathbf{v}}_{A} \cdot(\boldsymbol{\omega} \times \mathbf{a})$.

With the use of the functions $D\left(\theta, \omega_{3}\right), \tilde{E}\left(\theta, \dot{\theta}, \dot{\varphi}, \omega_{3}\right)$ we can write the TT equations of motion $(3)-(7)$ in an equivalent integrated form $[12,15]$ as

$$
\begin{aligned}
& \frac{d}{d t} \lambda\left(\theta, \dot{\theta}, \dot{\varphi}, \omega_{3}\right)=0, \\
& \frac{d}{d t} D\left(\theta, \omega_{3}\right)=\frac{\gamma m}{\alpha \sqrt{d(\hat{z} \cdot \hat{\mathbf{3}})}}(\hat{z} \times \mathbf{a}) \cdot \dot{\mathbf{v}}_{A}, \\
& \frac{d}{d t} \tilde{E}\left(\theta, \dot{\theta}, \dot{\varphi}, \omega_{3}\right)=m(\boldsymbol{\omega} \times \mathbf{a}) \cdot \dot{\mathbf{v}}_{A}, \\
& \frac{d}{d t} m \dot{\mathbf{r}}=-\mu g_{n} \mathbf{v}_{A} .
\end{aligned}
$$

These equations are as difficult as the equations (3)-(7). However, if we treat $D\left(\theta(t), \omega_{3}(t)\right)=$ : $D(t), \tilde{E}\left(\theta(t), \dot{\theta}(t), \dot{\varphi}(t), \omega_{3}(t)\right)=: \tilde{E}(t)$ as given known functions, then from $D(t)=I_{3} \omega_{3} \sqrt{d(\cos \theta)}$ and $\lambda=R I_{1} \dot{\varphi} \sin ^{2} \theta-R I_{3} \omega_{3}(\alpha-\cos \theta)$ we can calculate $\dot{\varphi}, \omega_{3}$ and substitute into expression (12) for the modified energy to obtain the METT $[12,15]$ that involves only the function $\theta(t)$ :

$$
\tilde{E}(t)=g(\cos \theta) \dot{\theta}^{2}+V(\cos \theta, D(t), \lambda) .
$$

This equation has the same form as equation (11), but now it depends explicitly on time through the functions $D(t)$ and $\tilde{E}(t)$. Solving this equation is therefore not longer possible. It is a first order time dependent ODE which we can study provided that we have some quantitative information about the functions $D(t)$ and $\tilde{E}(t)$. The functions $D(t), \tilde{E}(t)$ are usually unknown but for inverting solutions we have qualitative information about their behaviour due to conservation of the Jellett function $\lambda$.

Thus we consider the motion of the TT as being determined by the three functions $(\lambda, D(t)$, $\tilde{E}(t))$ and governed by the METT.

Of particular interest regarding the inversion movement is the initial and final position of the TT. The TT goes (asymptotically) from an initial angle close to $\theta=0$ to the final angle close to $\theta=\pi$ which means, since $\lambda=-\mathbf{L} \cdot \mathbf{a}$ is constant, that $\lambda=L_{0} R(1-\alpha)=L_{1} R(1+\alpha)$ (where $L_{0}$ and $L_{1}$ are the values of $|\mathbf{L}|$ at $\theta=0$ and $\theta=\pi$, respectively). This implies that $D_{0}=L_{0} \sqrt{d(1)}=\frac{\lambda}{R(1-\alpha)} \sqrt{\gamma+\sigma(1-\alpha)^{2}}$ and $D_{1}=-L_{1} \sqrt{d(-1)}=-\frac{\lambda}{R(1+\alpha)} \sqrt{\gamma+\sigma(1+\alpha)^{2}}$, and also that $\tilde{E}_{0}=\frac{\lambda^{2}}{2 R^{2} I_{3}(1-\alpha)^{2}}+m g R(1-\alpha)$ and $\tilde{E}_{1}=\frac{\lambda^{2}}{2 R^{2} I_{3}(1+\alpha)^{2}}+m g R(1+\alpha)$ [14]. 
The values $\left(D_{0}, \tilde{E}_{0}\right)$ and $\left(D_{1}, \tilde{E}_{1}\right)$ can be interpreted as the boundary values for the unknown functions $(D(t), \tilde{E}(t))$. So we assume that for inverting solutions $(D(t), \tilde{E}(t)) \stackrel{t \rightarrow \infty}{\longrightarrow}\left(D_{1}, \tilde{E}_{1}\right)$ and $(D(t), \tilde{E}(t)) \stackrel{t \rightarrow-\infty}{\longrightarrow}\left(D_{0}, \tilde{E}_{0}\right)$.

The aim of the following sections is to analyse dynamical properties of the inverting solution as the symmetry axis of TT moves from a neighborhood of $\theta=0$ to a neighborhood of $\theta=\pi$.

In order to simplify the technical side of analysis we choose special values of the parameters in METT so that the effective potential $V(\cos \theta, D, \lambda)$ becomes rational, but we expect that the whole line of reasoning can be repeated in the general case when the potential depends algebraically on $z$ through $\sqrt{d(z)}$.

We show that $V(z, D, \lambda)$ is strictly convex and therefore has one minimum $z_{\text {min }}$. We show also that, for inverting solutions when $D(t)$ moves from $D_{0}$ to $D_{1}$, the potential deforms so that $z_{\min }=z_{\min }(D, \lambda)$ moves from a neighborhood of $z=1$ to a neighborhood of $z=-1$.

Thus as the potential $V(z, D(t), \lambda)$ deforms and the modified energy $\tilde{E}(t)$ changes from $\tilde{E}_{0}$ to $\tilde{E}_{1}$ the angle $\theta(t)$ performs oscillatory motions between two turning angles $\theta_{ \pm}(t)$ satisfying the equation $V\left(\cos \theta_{ \pm}, D(t), \lambda\right)=\tilde{E}(t)$.

On the unit sphere $S^{2}$ the angle $\theta(t)$ performs nutational motion within the nutational band $\left[\theta_{-}(t), \theta_{+}(t)\right]$ that moves from the neighborhood of the north pole to the neighborhood of the south pole.

We shall give an estimate for the relation between the inversion time $T_{\text {inv }}$ and the maximal period of nutation $T_{V}(\tilde{E}(t), D(t))$, so that if $T_{\text {inv }}$ is an order of magnitude larger than $T_{V}$, say $T_{\text {inv }}>10 T_{V}$, the angle $\theta(t)$ performs oscillatory motion within the moving nutational band.

\subsection{The rational form of the METT}

The effective potential in the separation equation (11) is an algebraic function in $z$, which complicates the analysis. We can however make a restriction on the parameters so that the second degree polynomial $d(z)$ can be written as a perfect square. This makes the term $\sqrt{d(z)}$ a linear function of $z$ and the potential becomes a rational function $[2,16]$.

We see that if $1-\alpha^{2}<\gamma<1$, and if we let the parameter $\sigma=\frac{1-\gamma}{\gamma+\alpha^{2}-1}>0$, then $d(z)=$ $\gamma+\sigma(\alpha-z)^{2}+\sigma \gamma\left(1-z^{2}\right)=\frac{(\alpha-(1-\gamma) z)^{2}}{\gamma+\alpha^{2}-1}$. This is a perfect square, so for $\gamma$ in this range we can find physical values for $\sigma$ such that $\sqrt{d(z)}$ is a real polynomial in $z: \frac{\alpha-(1-\gamma) z}{\sqrt{\gamma+\alpha^{2}-1}}$. Note that $\left(1-\alpha^{2}, 1\right)$ is a subinterval of $(1-\alpha, 1+\alpha)$, the parameter range for $\gamma$ where complete inversion of TT is possible.

When $\sigma=\frac{1-\gamma}{\gamma+\alpha^{2}-1}$, we can rewrite the functions in the separation equation $E=g(\cos \theta) \dot{\theta}^{2}+$ $V(\cos \theta, D, \lambda)$ as

$$
\left.g(z)=\frac{I_{3}}{2}\left(\sigma\left(1+\alpha^{2}-2 \alpha z\right)+\gamma\right)=\frac{I_{3}}{2} \frac{1}{\gamma+\alpha^{2}-1}\left(\alpha^{2}+(1-\gamma)^{2}-2 \alpha(1-\gamma) z\right)\right),
$$

and

$$
\begin{aligned}
V(z, D, \lambda)= & m g R(1-\alpha z)+\frac{\left(\lambda(\alpha-(1-\gamma) z)+R D \sqrt{\gamma+\alpha^{2}-1}(\alpha-z)\right)^{2}}{2 I_{3} R^{2} \gamma^{2}\left(\gamma+\alpha^{2}-1\right)\left(1-z^{2}\right)} \\
& +\frac{R^{2} D^{2}\left(\gamma+\alpha^{2}-1\right)-(1-\gamma) \lambda^{2}}{2 R^{2} I_{1}\left(\gamma+\alpha^{2}-1\right)}
\end{aligned}
$$

This rational form of the effective potential is simpler to work with.

We should note that the restriction on the parameter $\sigma$ implies that the moments of inertia $I_{1}$ and $I_{3}$ are dependent on each other; if $\sigma=\frac{1-\gamma}{\gamma+\alpha^{2}-1}$ then $I_{1}=\frac{I_{3}^{2}+m R^{2} I_{3}\left(1-\alpha^{2}\right)}{I_{3}+m R^{2}}<I_{3}$. 


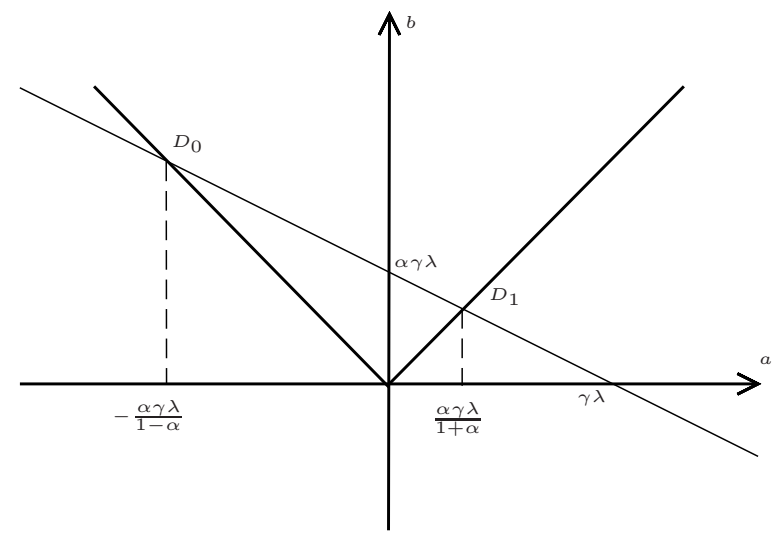

Figure 2. Linear relationship between the parameters $a$ and $b$. This line intersects the lines $b=a$ and $b=-a$ when $D=D_{1}$ and $D=D_{0}$, respectively.

\section{Convexity of the rational potential $V(z, D, \lambda)$}

The range of parameters making the effective potential a rational function provides a simplest non-trivial situation in which we can study properties of the potential in greater detail.

We consider the potential function

$$
\begin{aligned}
\tilde{V}(z, D, \lambda) & =-m g R \alpha z+\frac{\left(\lambda(\alpha-(1-\gamma) z)+R D \sqrt{\gamma+\alpha^{2}-1}(\alpha-z)\right)^{2}}{2 I_{3} R^{2} \gamma^{2}\left(\gamma+\alpha^{2}-1\right)\left(1-z^{2}\right)} \\
& =: \frac{1}{2 I_{3} R^{2} \gamma^{2}\left(\gamma+\alpha^{2}-1\right)}\left(-\beta z+\frac{(a z+b)^{2}}{1-z^{2}}\right)
\end{aligned}
$$

where $\tilde{V}(z, D, \lambda)=V(z, D, \lambda)-\left(m g R+\frac{R^{2} D^{2}\left(\gamma+\alpha^{2}-1\right)-(1-\gamma) \lambda^{2}}{2 R^{2} I_{1}\left(\gamma+\alpha^{2}-1\right)}\right)$, since the constant does not affect the shape of $V(z, D, \lambda)$ and the position of minimum $z_{\min }$. The parameters in the function $f(z)=-\beta z+\frac{(a z+b)^{2}}{1-z^{2}}$ (the expression inside the parentheses on the r.h.s. of $\left.(14)\right)$ are therefore defined as

$$
\begin{aligned}
& a=-(1-\gamma) \lambda-R D \sqrt{\gamma+\alpha^{2}-1} \\
& b=\alpha \lambda+\alpha R D \sqrt{\gamma+\alpha^{2}-1} \\
& \beta=2 m g R^{3} \alpha I_{3} \gamma^{2}\left(\gamma+\alpha^{2}-1\right) .
\end{aligned}
$$

Remember that $1-\alpha^{2}<\gamma<1$ and the range of parameters $a, b$ is determined by the range of $D$. We observe that $a+b=0$ if $D=D_{0}=\frac{\lambda}{R(1-\alpha)} \frac{(\alpha-1+\gamma)}{\sqrt{\gamma+\alpha^{2}-1}}$ and $a-b=0$ if $D=D_{1}=$ $-\frac{\lambda}{R(1+\alpha)} \frac{(\alpha+1-\gamma)}{\sqrt{\gamma+\alpha^{2}-1}}$. The parameters $a$ and $b$ satisfy the relation $b+\alpha a=\lambda \gamma \alpha$. It is illustrated in Fig. 2, where the lines $b=a$ and $b=-a$ correspond to $D=D_{1}$ and $D=D_{0}$, respectively.

Proposition 1. The effective potential $V(z, D, \lambda)$ of $(13)$ is convex for $z \in(-1,1)$ and for all real values of $D$ and $\lambda$.

Proof. We must show that $\frac{d^{2}}{d z^{2}} V(z, D, \lambda) \geq 0$ for $z \in(-1,1)$. Due to the form of $V$, it is enough to show that the rational function $\frac{(a z+b)^{2}}{1-z^{2}}$ is convex for all $a, b$. Suppose first $a \neq \pm b$ and $a b \neq 0$. We look at the second derivative of this function:

$$
\frac{d^{2}}{d z^{2}} \frac{(a z+b)^{2}}{1-z^{2}}=\frac{2}{\left(1-z^{2}\right)^{3}}\left(2 a b z^{3}+3\left(a^{2}+b^{2}\right) z^{2}+6 a b z+a^{2}+b^{2}\right)=\frac{2 q(z)}{\left(1-z^{2}\right)^{3}},
$$


and have to show that the third degree polynomial $q(z)=2 a b z^{3}+3\left(a^{2}+b^{2}\right) z^{2}+6 a b z+a^{2}+b^{2}$, has no roots in the interval $[-1,1]$.

To do this we apply the Sturm theorem [17]. We generate a sequence of polynomials $\left(q_{0}(z), q_{1}(z), q_{2}(z), \ldots, q_{m}(z)\right)$ recursively by starting from a square-free polynomial $q(z): q_{0}(z)=$ $q(z), q_{1}(z)=q^{\prime}(z)$ and $q_{i}=-\operatorname{rem}\left(q_{i-1}, q_{i-2}\right)$ for $i \geq 2$. Here $\operatorname{rem}\left(q_{i-1}, q_{i-2}\right)$ denotes the remainder after polynomial division of $q_{i-1}$ by $q_{i-2}$. By Euclid's algorithm, this will terminate with the constant polynomial $q_{m}$. Let $S(\xi)$ be the number of sign-changes in the sequence $\left(q_{0}(\xi)\right.$, $\left.q_{1}(\xi), q_{2}(\xi), \ldots, q_{m}(\xi)\right)$ at the point $\xi$. The Sturm theorem states that for real numbers $c<d$ the number of distinct roots in $(c, d]$ is $S(c)-S(d)$.

For our polynomial $q(z)$ the algorithm described above yields four polynomials $\left(q_{0}, q_{1}, q_{2}, q_{3}\right)$. When we look at this sequence of polynomials at the points $z=-1$ and $z=1$ we have

$$
\begin{aligned}
q_{0}(-1) & =4(a-b)^{2}, & q_{0}(1) & =4(a+b)^{2}, \\
q_{1}(-1) & =-6(a-b)^{2}, & q_{1}(1) & =6(a+b)^{2}, \\
q_{2}(-1) & =-\frac{\left(a^{2}-b^{2}\right)^{2}}{a b}, & q_{2}(1) & =\frac{\left(a^{2}-b^{2}\right)^{2}}{a b}, \\
q_{3}(-1) & =-6 a b, & q_{3}(1) & =-6 a b .
\end{aligned}
$$

We thus see that the number of sign changes for the Sturm sequence at both points -1 and 1 is the same, either 1 or 2 , depending on whether $a b$ is positive or negative. Thus according to Sturm's theorem, $q(z)$ has no roots in $(-1,1]$. Since $q(0)=a^{2}+b^{2}>0$ (and $\left.q(-1)>0\right), q(z)$ is positive in $[-1,1]$ and we can conclude that $\frac{(a z+b)^{2}}{1-z^{2}}$ is convex when $a \neq \pm b$ and $a b \neq 0$. Routine checking confirms that this is also convex if $a= \pm b$ and as well as if $a b=0$. Thus the function is convex for all arbitrary values of the parameters $a, b \in \mathbb{R}$.

\subsection{Estimates for position of minimum of $V(z, D, \lambda)$}

The result that $V(z, D, \lambda)$ is convex for $z \in(-1,1)$, combined with $V(z, D, \lambda) \rightarrow \infty$ as $z \rightarrow \pm 1$, gives that the potential will have exactly one minimum $z_{\min }$ in $[-1,1]$. We use this to analyse how this minimum shifts as $D$ goes from $D_{0}$ to $D_{1}$, for $\lambda>\lambda_{\text {thres. }}$. We show below that for every (small) $\epsilon>0$ we can find $\delta_{+}(\epsilon, \lambda)>0$ and $\delta_{-}(\epsilon, \lambda)>0$ such that for $0<\delta<\delta_{+}(\epsilon, \lambda)$ and $0<\delta<\delta_{-}(\epsilon, \lambda)$ the potential $V\left(z, D_{0} \mp \delta /\left(R(1-\alpha) \sqrt{\gamma+\alpha^{2}-1}\right), \lambda\right)$ has a minimum in $[1-\epsilon, 1]$ and $V\left(z, D_{1} \pm \delta /\left(R(1+\alpha) \sqrt{\gamma+\alpha^{2}-1}\right), \lambda\right)$ has a minimum in $[-1,-1+\epsilon]$.

This means that as the inclination angle $\theta$ goes from a value close to 0 to a value close to $\pi$ and $D(t)$ goes from a neighborhood $\left(D_{0}-\frac{\delta}{R(1-\alpha) \sqrt{\gamma+\alpha^{2}-1}}, D_{0}+\frac{\delta}{R(1-\alpha) \sqrt{\gamma+\alpha^{2}-1}}\right)$ to a neighborhood $\left(D_{1}-\frac{\delta}{R(1+\alpha) \sqrt{\gamma+\alpha^{2}-1}}, D_{1}+\frac{\delta}{R(1+\alpha) \sqrt{\gamma+\alpha^{2}-1}}\right)$, the minimum of $V(\cos \theta, D, \lambda)$ moves from a neighborhood of $\theta=0$ to a neighborhood of $\theta=\pi$.

As explained before, it is sufficient to study the position of $z_{\min }$ for the function $2 I_{3} R^{2} \gamma^{2}(\gamma+$ $\left.\alpha^{2}-1\right) \tilde{V}(z, D(a, b), \lambda(a, b))=: f(z)$ with

$$
f(z)=\frac{(a z+b)^{2}}{1-z^{2}}-\beta z
$$

The derivative is $f^{\prime}(z)=\frac{p(z)}{\left(1-z^{2}\right)^{2}}$, where $p(z)=2(a z+b)(b z+a)-\beta\left(1-z^{2}\right)^{2}$.

Our question about the position of $z_{\min }$ can be reduced to the question: for which values of the parameters $a, b, \beta$ does the polynomial $p(z)$ have one zero in one of the subintervals $[1-\epsilon, 1]$ and $[-1,-1+\epsilon]$ of the interval $[-1,1]$ ?

We see that $p(-1)=-2(a-b)^{2}$ and $p(1)=2(a+b)^{2}$, which confirms that $f^{\prime}(z)$ has a zero and changes sign in $[-1,1]$ (remember that $a \neq \pm b$ if $D \neq D_{1}$ and $D \neq D_{0}$ ). 
Proposition 2. Assume that $\lambda>\lambda_{\text {thres }}=\frac{\sqrt{m g R^{3} I_{3} \alpha}(1+\alpha)^{2}}{\sqrt{1+\alpha-\gamma}}$.

i) For any $($ small $) \epsilon>0$ there is $\delta_{-}(\epsilon, \lambda)>0$ being a positive solution of the equation

$$
\alpha(1-\alpha) \gamma \lambda \delta+\alpha \delta^{2}+\frac{\delta^{2}}{\epsilon^{2}}(1-\epsilon)(1+\alpha)^{2}=\alpha^{2} \gamma^{2} \lambda^{2}-\frac{\beta}{2}(2-\epsilon)^{2}(1+\alpha)^{2},
$$

such that for every positive $\delta<\delta_{-}(\epsilon, \lambda)$ the potential $V(z, D, \lambda)$ has a minimum $z_{\min }$ in the interval $[-1,-1+\epsilon]$ for $D=D_{1} \pm \frac{\delta}{R(1+\alpha) \sqrt{\gamma+\alpha^{2}-1}}$.

ii) For any $($ small $) \epsilon>0$ there is $\delta_{+}(\epsilon, \lambda)=\min \left\{\delta_{1}, \delta_{2}\right\}>0$, where $\delta_{1}=\gamma(1+\alpha) \lambda$ and $\delta_{2}$ is the positive solution of the equation

$$
\frac{\delta^{2}}{\epsilon^{2}}(1-\epsilon)(1-\alpha)^{2}+\alpha \gamma \lambda \delta(1+\alpha)-\alpha \delta^{2}=\alpha^{2} \gamma^{2} \lambda^{2}+\frac{\beta}{2}(2-\epsilon)^{2}(1-\alpha)^{2},
$$

such that for every positive $\delta<\delta_{+}(\epsilon, \lambda)$ the potential $V(z, D, \lambda)$ has a minimum $z_{\min }$ in the interval $[1-\epsilon, 1]$ for $D=D_{0} \mp \frac{\delta}{R(1-\alpha) \sqrt{\gamma+\alpha^{2}-1}}$.

Proof. We know that $V(z, D, \lambda)$ has one minimum in $[-1,1]$ and $p(z)$, the numerator of the derivative $f^{\prime}(z)$, has one zero in $[-1,1]$. The question is to formulate conditions for the parameter $D$ so that this zero is located in the interval $[-1,-1+\epsilon]$ or $[1-\epsilon, 1]$.

i) We have to show that for any (small) $\epsilon>0$ and $\lambda>\lambda_{\text {thres }}$ there is $\delta_{-}(\epsilon, \lambda)>0$ such that for any $\delta<\delta_{-}(\epsilon, \lambda)$ the potential $V\left(z, D=D_{1} \pm \frac{\delta}{R(1+\alpha) \sqrt{\gamma+\alpha^{2}-1}}, \lambda\right)$ has a minimum in the interval $[-1,-1+\epsilon]$. To find this $\delta_{-}(\epsilon, \lambda)$ we have to study the signs of $p(-1)=-2(a-b)^{2}<0$ and of

$$
p(-1+\epsilon)=p(-1)+2(a-b)^{2} \epsilon+2(a b-2 \beta) \epsilon^{2}+4 \beta \epsilon^{3}-\beta \epsilon^{4} .
$$

To have minimum $z_{\min }$ in $[-1,-1+\epsilon]$ we need to have $p(-1+\epsilon)>0$. If $D=D_{1} \pm \frac{\delta}{R(1+\alpha) \sqrt{\gamma+\alpha^{2}-1}}$ then $(a-b)^{2}=\delta^{2}, a b=\frac{1}{(1+\alpha)^{2}}(\alpha \gamma \lambda-\delta)(\alpha \gamma \lambda+\alpha \delta)$ and

$$
\begin{aligned}
p(-1+\epsilon) & =-2 \delta^{2}+2 \delta^{2} \epsilon+2 a b \epsilon^{2}-\beta \epsilon^{2}(2-\epsilon)^{2} \\
& =-2 \delta^{2}(1-\epsilon)+\frac{2 \epsilon^{2}}{(1+\alpha)^{2}}\left(\alpha^{2} \gamma^{2} \lambda^{2}-\alpha \gamma(1-\alpha) \delta \lambda-\alpha \delta^{2}\right)-\beta \epsilon^{2}(2-\epsilon)^{2} .
\end{aligned}
$$

For positivity of $p(-1+\epsilon)$ we must have

$$
\alpha(1-\alpha) \gamma \lambda \delta+\alpha \delta^{2}+\frac{\delta^{2}}{\epsilon^{2}}(1-\epsilon)(1+\alpha)^{2}<\alpha^{2} \gamma^{2} \lambda^{2}-\frac{\beta}{2}(2-\epsilon)^{2}(1+\alpha)^{2} .
$$

The r.h.s. of (18) is positive since

$$
(\alpha \gamma \lambda)^{2}-\frac{\beta}{2}(2-\epsilon)^{2}(1+\alpha)^{2}=(\alpha \gamma \lambda)^{2}\left[1-\frac{2 \beta}{\lambda^{2}} \frac{(1+\alpha)^{2}}{\alpha^{2} \gamma^{2}} \frac{(2-\epsilon)^{2}}{4}\right],
$$

and for $\lambda=C \lambda_{\text {thres }}, C>1$, the term in the square brackets can be shown to be positive. We see this when we write

$$
\frac{2 \beta}{\lambda^{2}} \frac{(1+\alpha)^{2}}{\alpha^{2} \gamma^{2}} \frac{(2-\epsilon)^{2}}{4}=4\left[\frac{\left(\gamma+\alpha^{2}-1\right)(1+\alpha-\gamma)}{\alpha^{2}(1+\alpha)^{2}}\right] \frac{1}{C^{2}} \frac{(2-\epsilon)^{2}}{4} .
$$


Clearly $\frac{1}{C^{2}} \frac{(2-\epsilon)^{2}}{4}<1$ and the function $\rho(\alpha, \gamma)=\frac{\left(\gamma+\alpha^{2}-1\right)(1+\alpha-\gamma)}{\alpha^{2}(1+\alpha)^{2}}<\frac{1}{4}$ for $1-\alpha^{2}<\gamma<1,0<$ $\alpha<1$. So the r.h.s. of (18) is indeed positive. The 1.h.s. of (18) has the form $\delta[\alpha(1-\alpha) \gamma \lambda+$ $\left.\alpha \delta+\frac{\delta}{\epsilon^{2}}(1-\epsilon)(1+\alpha)^{2}\right]$ with all terms positive. If we define $\delta_{-}(\epsilon, \lambda)$ as the positive solution of

$$
\alpha(1-\alpha) \gamma \lambda \delta+\alpha \delta^{2}+\frac{\delta^{2}}{\epsilon^{2}}(1-\epsilon)(1+\alpha)^{2}=\alpha^{2} \gamma^{2} \lambda^{2}-\frac{\beta}{2}(2-\epsilon)^{2}(1+\alpha)^{2},
$$

then for all positive $\delta<\delta_{-}(\epsilon, \lambda)$ we have $p(-1+\epsilon)>0$ and the minimum $z_{\min } \in[-1,-1+\epsilon]$.

ii) We have to show that for any (small) $\epsilon>0$ and $\lambda>\lambda_{\text {thres }}$ there is $\delta_{+}(\epsilon, \lambda)>0$ such that for any positive $\delta<\delta_{+}(\epsilon, \lambda)$ the potential $V\left(z, D=D_{0} \mp \frac{\delta}{R(1-\alpha) \sqrt{\gamma+\alpha^{2}-1}}\right)$ has a minimum $z_{\text {min }}$ in the interval $[1-\epsilon, 1]$. To find this $\delta_{+}(\epsilon, \lambda)$ we need to study the signs of $p(1)=2(a+b)^{2}>0$ and of

$$
p(1-\epsilon)=p(1)-2(a+b)^{2} \epsilon+2(a b-2 \beta) \epsilon^{2}+\beta \epsilon^{3}(4-\epsilon) .
$$

To have minimum $z_{\min }$ in $[1-\epsilon, 1]$ we need to show how to find $\delta$ so that $p(1-\epsilon)<0$. If $D=D_{0} \mp \frac{\delta}{R(1-\alpha) \sqrt{\gamma+\alpha^{2}-1}}$ then $(a+b)^{2}=\delta^{2}, a b=-\frac{1}{(1-\alpha)^{2}}(\alpha \gamma \lambda-\delta)(\alpha \gamma \lambda-\alpha \delta)$ and

$$
p(1-\epsilon)=2 \delta^{2}(1-\epsilon)-\frac{2 \epsilon^{2}}{(1-\alpha)^{2}}\left((\alpha \gamma \lambda-\delta)(\alpha \gamma \lambda-\alpha \delta)+2 \beta(1-\alpha)^{2}\right)+\beta \epsilon^{3}(4-\epsilon) .
$$

For negativity of $p(1-\epsilon)$ we must have

$$
\frac{\delta^{2}}{\epsilon^{2}}(1-\epsilon)(1-\alpha)^{2}+\alpha \gamma \lambda \delta(1+\alpha)-\alpha \delta^{2}<\alpha^{2} \gamma^{2} \lambda^{2}+\frac{\beta}{2}(2-\epsilon)^{2}(1-\alpha)^{2} .
$$

The r.h.s. of (21) is obviously positive. The l.h.s. of (21) has the form

$$
\delta\left[\frac{\delta}{\epsilon^{2}}(1-\epsilon)(1-\alpha)^{2}+\alpha((1+\alpha) \gamma \lambda-\alpha \delta)\right]
$$

which is certainly positive when $\lambda>\frac{\delta}{\gamma(1+\alpha)}$ since $\delta>0$ can always be chosen so small that this is true, by the chosen convention $\lambda>0$. If we define $\delta_{+}(\epsilon, \lambda)=\min \left\{\delta_{1}, \delta_{2}\right\}$, where $\delta_{1}=\gamma(1+\alpha) \lambda$ and $\delta_{2}$ is the positive solution of

$$
\frac{\delta^{2}}{\epsilon^{2}}(1-\epsilon)(1-\alpha)^{2}+\alpha \gamma \lambda \delta(1+\alpha)-\alpha \delta^{2}=\alpha^{2} \gamma^{2} \lambda^{2}+\frac{\beta}{2}(2-\epsilon)^{2}(1-\alpha)^{2},
$$

then for all positive $\delta<\delta_{+}(\epsilon, \lambda)$ we have $p(1-\epsilon)<0$ and the minimum $z_{\min } \in[1-\epsilon, 1]$.

Example 1. We exemplify the above proposition for some physical values for the parameters. We let $m=0.02 \mathrm{~kg}, R=0.02 \mathrm{~m}$ and $\alpha=0.3$. We shall assume $I_{3}=\frac{2}{5} m R^{2}$, and we get a rational potential (13) when $I_{1}=\frac{I_{3}\left(I_{3}+m R^{2}\left(1-\alpha^{2}\right)\right)}{I_{3}+m R^{2}}=\frac{131}{350} m R^{2}$, which means that $\gamma=\frac{131}{140} \in$ $\left(1-\alpha^{2}, 1\right)=(0.91,1)$. Jellett's integral $\lambda$ will for simplicity be assumed to be two times the threshold value $\lambda_{\text {thres }}$, so $\lambda=6.88 \cdot 10^{-6} \mathrm{~kg} \cdot \mathrm{m}^{3} \cdot \mathrm{s}^{-1}$.

Suppose first that $\epsilon=0.1$ and consider the condition for a minimum $z_{\min }$ to stay in the interval $(-1,-1+\epsilon)$. We have $D_{1}=-6 \cdot 10^{-4}$ and by solving $(20)$ we get $\delta_{-}=1.48 \cdot 10^{-7}$, so if $D=D_{1}+\delta$, with $\delta<\frac{\delta_{-}}{R(1+\alpha) \sqrt{\gamma+\alpha^{2}-1}} \approx 3.5 \cdot 10^{-5}$, the potential has its minimum in the interval.

If $\epsilon$ is reduced to 0.01 , the bound for $\delta$ is tightened one order of magnitude as well. 


\section{Oscillation of $\theta(t)$ within the deforming rational potential $V(\cos \theta, D(t), \lambda)$}

As a toy TT inverts, we can see that the symmetry axis performs oscillations, or equivalently we say that it nutates. This is also apparent in simulations of the equations of motion $[3,11,18]$ where graphs of the evolution of the inclination angle $\theta(t)$ show that it rises in an oscillating manner from an angle close to $\theta=0$ to an angle close to $\theta=\pi$. Here we say that a solution $\theta(t)$ is oscillatory on a time interval $[0, T]$ when $\dot{\theta}(t)$ changes sign a number of times in this interval.

We consider solutions of METT with $D(t), \tilde{E}(t)$ describing inverting solutions of TT equations, under assumption that $D(t), \tilde{E}(t)$ are slowly varying regular functions moving from a small neighborhood of $\left(D_{0}, \tilde{E}_{0}\right)$ to a small neighborhood of $\left(D_{1}, \tilde{E}_{1}\right)$. They are regular since $D(t)=D\left(\theta(t), \omega_{3}(t)\right)$ and $\tilde{E}(t)=\tilde{E}\left(\theta(t), \dot{\theta}(t), \dot{\varphi}(t), \omega_{3}(t)\right)$. We further have assumed that $(D(t), \tilde{E}(t)) \stackrel{t \rightarrow \infty}{\longrightarrow}\left(D_{1}, \tilde{E}_{1}\right)$ and $(D(t), \tilde{E}(t)) \stackrel{t \rightarrow-\infty}{\longrightarrow}\left(D_{0}, \tilde{E}_{0}\right)$.

In the limiting case of constant $D$ and $\tilde{E}$ when the METT describes purely rolling TT, the oscillating behaviour of $\theta(t)$ follows from the dynamical system representation of the second order equation for $\theta(t)$. For the rolling TT the energy

$$
\tilde{E}=g(\cos \theta) \dot{\theta}^{2}+V(\cos \theta, D, \lambda)
$$

is an integral of motion for the $\theta$-equation obtained by differentiating (22):

$$
\ddot{\theta}=\frac{\sin \theta}{2 g(\cos \theta)}\left(g_{z}^{\prime}(\cos \theta) \dot{\theta}^{2}+V_{z}^{\prime}(\cos \theta, D, \lambda)\right)
$$

where $g(z=\cos \theta)=\frac{I_{3}\left(\alpha^{2}+(1-\gamma)^{2}-2 \alpha(1-\gamma) z\right)}{2\left(\gamma+\alpha^{2}-1\right)}$. This means that $\tilde{E}=g(\cos \theta) y^{2}+V(\cos \theta, D, \lambda)$ is an integral of motion for the dynamical system

$$
\begin{aligned}
& \dot{\theta}=y, \\
& \dot{y}=\frac{\sin \theta}{2 g(\cos \theta)}\left(g_{z}^{\prime}(\cos \theta) y^{2}+V_{z}^{\prime}(\cos \theta, D, \lambda)\right) .
\end{aligned}
$$

Trajectories of system (23) are lines of constant value of energy $\tilde{E}$ and they are closed curves. The closed trajectories describe periodic solutions [2] with period $T$ defined by the integral

$$
T(\tilde{E})=2 \int_{\theta_{1}}^{\theta_{2}} \frac{\sqrt{g(\cos \theta)} d \theta}{\sqrt{\tilde{E}-V(\cos \theta, D, \lambda)}},
$$

where the turning latitudes $\theta_{1}<\theta_{2}$ are defined by $\tilde{E}=V\left(\cos \theta_{1,2}, D, \lambda\right)$.

The modified energy $\tilde{E}(t)$ is bounded. That entails boundedness of $\boldsymbol{\omega}(t)=-\dot{\varphi} \sin \theta \hat{\mathbf{1}}+\dot{\theta} \hat{\mathbf{2}}+$ $(\dot{\psi}+\dot{\varphi} \cos \theta) \hat{\mathbf{3}}$ and thus $|\dot{\theta}(t)|<B$ for some positive $B$. Since the potential $V(z, D(t), \lambda) \stackrel{\theta \rightarrow 0, \pi}{\longrightarrow} \infty$ the curve $(\theta(t), \dot{\theta}(t))$ of each inverting solution is confined to the open rectangle $(\theta, \dot{\theta}) \in(0, \pi) \times$ $(-B, B)$.

The picture that emerges is that, for slowly varying $\tilde{E}(t), D(t)$, the inverting trajectories of METT stay (locally) close to the trajectories of system (23) and are traversed with almost the same velocity. The time of passing $T=t_{2}-t_{1}$ between two turning angles given by $V\left(\cos \theta_{1}, D\left(t_{1}\right), \lambda\right)=\tilde{E}\left(t_{1}\right)$ and $V\left(\cos \theta_{2}, D\left(t_{2}\right), \lambda\right)=\tilde{E}\left(t_{2}\right)$ is close to the half-period of the non-deforming potential.

Thus initially the trajectory moves around $\left(\theta_{\min }, \dot{\theta}=0\right)$, with $\theta_{\min }$ close to 0 . As $D(t) \rightarrow D_{1}$ the minimum $\theta_{\min }$ moves toward $\theta=\pi$ (see Proposition 2) and for sufficiently slowly varying $D(t)$ the trajectory goes several times around $\left(\theta_{\min }, \dot{\theta}=0\right)$ and drifts toward the point 

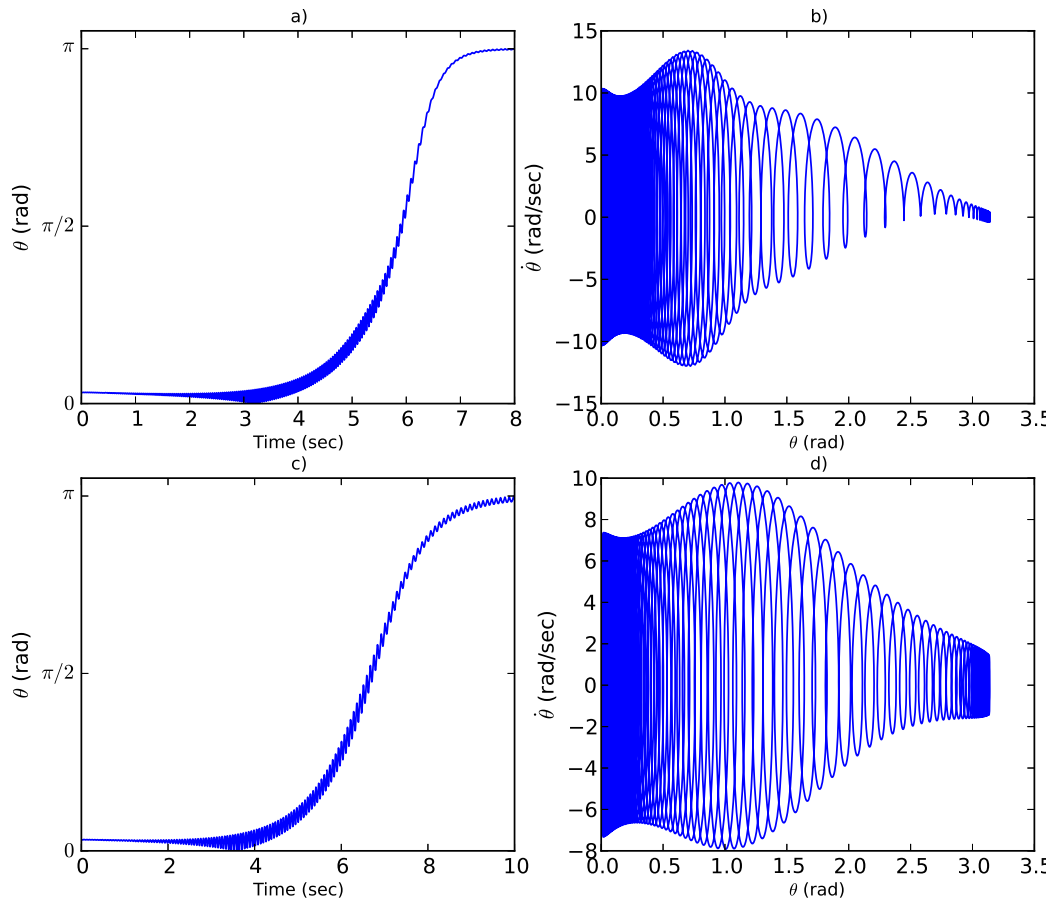

Figure 3. Plots of $(t, \theta(t))$ (left) and $(\theta(t), \dot{\theta}(t))$ (right) obtained by integrating equations (3)-(7) and (8) for two sets of parameters and initial values. Plots $a$ and $b$ correspond to parameter values for rational potential in Example 1 and $\mu=0.3$. Plots $c$ and $d$ correspond to parameter values provided in [3], corresponding to an algebraic potential. The equations are integrated using the Python 2.7 open source library SciPy [8].

$(\theta=\pi, \dot{\theta}=0)$. The trajectory describes a selfcrossing and contracting spiral with the center moving toward $(\pi, 0)$ in the $(\theta, \dot{\theta})$-plane. The solution curve for $\theta(t)$ is a superposition of a small amplitude oscillating component with a logistic type curve going from $\theta=0$ to $\theta=\pi$.

In Fig. 3 we see this. The plots $a$ and $b$ present a graph of $\theta(t)$ and a curve $(\theta(t), \dot{\theta}(t))$ obtained by solving equations of motion (3)-(7) for the rolling and gliding TT, with $g_{n}$ given by equation (8) and with values of parameters taken from Example 1. So the effective potential $V$ given by (13) is rational. We have taken $\mu=0.3$ and we have integrated the equations numerically for initial values $\theta(0)=0.1 \mathrm{rad}, \dot{\theta}(0)=\dot{\varphi}(0)=0, \nu_{x}(0)=\nu_{y}(0)=0$ and $\omega_{3}(0)=155.0 \mathrm{rad} / \mathrm{s}$ (corresponding to the value of $\lambda=2 \lambda_{\text {thres }}$ ).

Plot $a$ shows oscillations of $\theta(t)$ as it rises to $\pi$. The time of inversion from the moment when $\theta(t)$ starts to rise, at approximately 3 seconds, is about $4-5$ seconds. Larger values of $\mu$ give shorter time of inversion. Plot $b$ shows the trajectory in the $(\theta, \dot{\theta})$-plane.

In Fig. $3 c$ and $d$ the same type of numerical integration is performed, for parameters giving an algebraic (not rational) effective potential. The parameters are the same as used in Cohen [3] and they are close to values taken in Example 1. Here $m=0.015 \mathrm{~kg}, R=0.025 \mathrm{~m}, \alpha=0.2$, $I_{1}=I_{3}=\frac{2}{5} m R^{2}$ and $\mu=0.3$ with initial values $\theta(0)=0.1 \mathrm{rad}, \dot{\theta}(0)=\dot{\varphi}(0)=0, \nu_{x}(0)=$ $\nu_{y}(0)=0$ and $\omega_{3}(0)=100 \mathrm{rad} / \mathrm{s}$. We may note that Cohen uses a Coulomb-type gliding friction force, so the equations solved in his article are slightly different than equations (3)-(7).

In the next section we shall estimate the maximum value of the period of oscillations for all values of the modified energy $\tilde{E}$ and $D$.

This will allow us to formulate a condition for the time of inversion needed for oscillatory behaviour of $\theta(t)$. Basically $T_{\text {inv }}$ has to be an order of magnitude larger than $T_{\text {upp }}$ - the maximal period of oscillations within potential $V(\cos \theta, D(t), \lambda)$. 


\subsection{Estimates for the period of oscillation}

A direct way of estimating the period of nutation is to study the explicit integral defining the period and expanding it w.r.t. a small parameter $\epsilon=\frac{2 \beta}{b^{2}}$, with $b$ and $\beta$ given by (16) and (17), respectively. This is the technique used in [16], but in that paper the analysis is based on the assumption that $\omega_{3}$ is large. Here we use the more general assumption that $\lambda$ is only above the threshold value, so that $\lambda=C \lambda_{\text {thres }}$ with $C>1$. To simplify estimates we also assume that we consider curves $(D(t), \tilde{E}(t))$ such that $D_{1}<D<D_{0}$. Then by using that $b=\alpha \lambda+\alpha R D \sqrt{\gamma+\alpha^{2}-1}$ satisfies $\frac{\alpha \gamma \lambda}{1+\alpha}<b<\frac{\alpha \gamma \lambda}{1-\alpha}$ for $D_{1}<D<D_{0}$, we have the estimate

$$
\epsilon=\frac{2 \beta}{b^{2}}<\frac{2 \beta(1+\alpha)^{2}}{\lambda^{2} \alpha^{2} \gamma^{2}}=\frac{4}{C^{2}} \cdot\left[\frac{\left(\gamma+\alpha^{2}-1\right)(1+\alpha-\gamma)}{\alpha^{2}(1+\alpha)^{2}}\right]<\frac{4}{C^{2}} \cdot \frac{1}{4}<1,
$$

for $1-\alpha^{2}<\gamma<1,0<\alpha<1$, similar to the estimate for equation (19).

In the following we shall determine the dependence of the period $T(\epsilon)$ on the small parameter $\epsilon=\frac{2 \beta}{b^{2}}$ and we shall find an estimate for the maximal value of $T(\epsilon)$ that is valid for all $D \in$ $\left(D_{1}, D_{0}\right)$ and all values $\epsilon<1$, which means all values of the Jellett integral that are above the threshold value. As known from the asymptotic analysis these initial values of $\lambda$ lead to inversion of the TT.

The period of oscillations for the potential $V(z, D, \lambda)$ is given by the integral

$$
T=2 \int_{z_{1}}^{z_{2}} \frac{\sqrt{g(z)} d z}{\sqrt{\left(1-z^{2}\right)(\tilde{E}-V(z, D, \lambda))}}
$$

where $z_{1}<z_{2} \in(-1,1)$ are two turning points defined by $\tilde{E}=V\left(z_{1,2}, D, \lambda\right)$. This equation always has two solutions for $\tilde{E}>V\left(z_{\min }, D, \lambda\right)$ since the potential is convex and

$$
\lim _{z \rightarrow \pm 1} V(z, D, \lambda)=\infty
$$

In terms of the the parameters $a$ and $b$, the potential (13) reads

$$
V(z, D, \lambda)=m g R(1-\alpha z)+\frac{(a z+b)^{2}}{2 I_{3} R^{2} \gamma^{2}\left(\gamma+\alpha^{2}-1\right)\left(1-z^{2}\right)}+\frac{\alpha^{2} a^{2}-(1-\gamma) b^{2}}{2 R^{2} \alpha^{2} I_{3} \gamma^{2}\left(\gamma+\alpha^{2}-1\right)},
$$

and (by definition) the left turning point $z_{1}$ is given implicitly by the equation

$$
\tilde{E}=m g R\left(1-\alpha z_{1}\right)+\frac{\left(a z_{1}+b\right)^{2}}{2 I_{3} R^{2} \gamma^{2}\left(\gamma+\alpha^{2}-1\right)\left(1-z_{1}^{2}\right)}+\frac{\alpha^{2} a^{2}-(1-\gamma) b^{2}}{2 R^{2} \alpha^{2} I_{3} \gamma^{2}\left(\gamma+\alpha^{2}-1\right)} .
$$

In the following we shall parametrise (similarly as in [16]) the remaining roots of $\left(1-z^{2}\right)(\tilde{E}-$ $V(z, D, \lambda))$ by $z_{1}$. Due to this they become solutions of a quadratic equation. The function in the denominator of (25) we write as

$$
\begin{aligned}
& \left(1-z^{2}\right)(\tilde{E}-V(z, D, \lambda))=\frac{1}{\left(1-z_{1}^{2}\right)}\left(-m g R \alpha\left(z_{1}-z\right)\left(1-z_{1}^{2}\right)\left(1-z^{2}\right)\right. \\
& \left.\quad+\frac{\left(a z_{1}+b\right)^{2}\left(1-z^{2}\right)-(a z+b)^{2}\left(1-z_{1}^{2}\right)}{2 I_{3} R^{2} \gamma^{2}\left(\gamma+\alpha^{2}-1\right)}\right) \\
& =\frac{\beta\left(z_{1}-z\right)}{2 I_{3} R^{2} \gamma^{2}\left(\gamma+\alpha^{2}-1\right)}\left(z^{2}+\frac{a^{2}+b^{2}+2 a b z_{1}}{\beta\left(1-z_{1}^{2}\right)} z-1+\frac{\left(a^{2}+b^{2}\right) z_{1}+2 a b}{\beta\left(1-z_{1}^{2}\right)}\right) .
\end{aligned}
$$


Notice that $z= \pm 1$ are not roots because $V(z, D, \lambda)$ has singularities at the points $z= \pm 1$. The quadratic polynomial in the parentheses of (26) determines roots $z_{2}, z_{3}$, for any given turning point $z_{1}$. They are thus solutions of the quadratic equation

$$
z^{2}+\left(\frac{a^{2}+b^{2}+2 a b z_{1}}{\beta\left(1-z_{1}^{2}\right)}\right) z-1+\frac{\left(a^{2}+b^{2}\right) z_{1}+2 a b}{\beta\left(1-z_{1}^{2}\right)}=0 .
$$

By $z_{3}$ we denote the root satisfying $z_{3}<-1$ and by $z_{2}\left(>z_{1}\right)$ the right turning point. The polynomial $\left(1-z^{2}\right)(E-V(z, D, \lambda))$ is then factorized:

$$
\left(1-z^{2}\right)(E-V(z, D, \lambda))=\frac{\beta\left(z_{1}-z\right)\left(z_{2}-z\right)\left(z_{3}-z\right)}{2 I_{3} R^{2} \gamma^{2}\left(\gamma+\alpha^{2}-1\right)}=m g R \alpha\left(z_{1}-z\right)\left(z_{2}-z\right)\left(z_{3}-z\right) .
$$

We substitute this into the integral for the period $T(25)$. By the mean value theorem for integrals there exist a $z^{*} \in\left[z_{1}, z_{2}\right]$ such that we have

$$
T=\frac{2 \sqrt{g\left(z^{*}\right)}}{\sqrt{m g R \alpha}} \int_{z_{1}}^{z_{2}} \frac{d z}{\sqrt{\left(z_{1}-z\right)\left(z_{2}-z\right)\left(z_{3}-z\right)}},
$$

where $g\left(z^{*}\right)=\frac{I_{3}\left(\alpha^{2}+(1-\gamma)^{2}-2 \alpha(1-\gamma) z^{*}\right)}{2\left(\gamma+\alpha^{2}-1\right)}$.

The integral can be transformed to a standard complete elliptic integral of the first kind through the change of variables $z=z_{2}+\left(z_{1}-z_{2}\right) s^{2}[6]$ :

$$
T=\frac{4 \sqrt{g\left(z^{*}\right)}}{\sqrt{m g R \alpha}} \frac{1}{\sqrt{z_{2}-z_{3}}} \int_{0}^{1} \frac{d s}{\sqrt{\left(1-s^{2}\right)\left(1-k^{2} s^{2}\right)}},
$$

where $k^{2}=\frac{z_{2}-z_{1}}{z_{2}-z_{3}}<1$ is a positive parameter. This integral has the standard expansion

$$
K\left(k^{2}\right)=\int_{0}^{1} \frac{d s}{\sqrt{\left(1-s^{2}\right)\left(1-k^{2} s^{2}\right)}}=\frac{\pi}{2} \sum_{n=0}^{\infty}\left(\frac{(2 n-1) ! !}{(2 n) ! !}\right)^{2} k^{2 n}=\frac{\pi}{2}\left(1+\frac{k^{2}}{4}+O\left(k^{4}\right)\right) .
$$

By using the roots of equation (27) and by expanding $k^{2}=\frac{z_{2}-z_{1}}{z_{2}-z_{3}}$ w.r.t. $\epsilon=\frac{2 \beta}{b^{2}}$, we can show that $k^{2}=O(\epsilon)$ if $\epsilon$ is small. Indeed, after solving equation (27) we get that the quantity $k^{2}$ can be written:

$$
\begin{aligned}
k^{2}= & \frac{z_{2}-z_{1}}{z_{2}-z_{3}}=\frac{1}{2}-\frac{1}{2}\left(z_{1}+\frac{a^{2}+b^{2}+2 a b z_{1}}{2 \beta\left(1-z_{1}^{2}\right)}\right) \\
& \times\left(\frac{\left(a^{2}+b^{2}+2 a b z_{1}\right)^{2}}{4 \beta^{2}\left(1-z_{1}^{2}\right)^{2}}+1-\frac{\left(a^{2}+b^{2}\right) z_{1}+2 a b}{\beta\left(1-z_{1}^{2}\right)}\right)^{-\frac{1}{2}} \\
= & \frac{1}{2}-\frac{1}{2}\left(z_{1} \frac{2 \beta\left(1-z_{1}^{2}\right)}{a^{2}+b^{2}+2 a b z_{1}}+1\right) \\
& \times\left(1+\frac{4 \beta^{2}\left(1-z_{1}^{2}\right)^{2}}{\left(a^{2}+b^{2}+2 a b z_{1}\right)^{2}}-\frac{4 \beta\left(1-z_{1}^{2}\right)\left(\left(a^{2}+b^{2}\right) z_{1}+2 a b\right)}{\left(a^{2}+b^{2}+2 a b z_{1}\right)^{2}}\right)^{-\frac{1}{2}} .
\end{aligned}
$$

With the parameters $\epsilon=\frac{2 \beta}{b^{2}}$ and $w=\frac{a}{b}$, the expansion of $k^{2}$ is:

$$
\begin{aligned}
k^{2}= & \frac{1}{2}-\frac{1}{2}\left(\frac{z_{1}\left(1-z_{1}^{2}\right)}{w^{2}+1+2 w z_{1}} \epsilon+1\right) \\
& \times\left(1+\frac{\left(1-z_{1}^{2}\right)^{2}}{\left(w^{2}+1+2 w z_{1}\right)^{2}} \epsilon^{2}-\frac{2\left(1-z_{1}^{2}\right)\left(\left(w^{2}+1\right) z_{1}+2 w\right)}{\left(w^{2}+1+2 w z_{1}\right)^{2}} \epsilon\right)^{-\frac{1}{2}}
\end{aligned}
$$




$$
=-\frac{\left(1+z_{1} w\right)\left(w+z_{1}\right)\left(1-z_{1}^{2}\right)}{\left(1+w^{2}+2 w z_{1}\right)^{2}} \epsilon+O\left(\epsilon^{2}\right) .
$$

It should be noted that the minus sign here is misleading. When (31) is expressed by parameters $D, \lambda$, one sees that the factor at $\epsilon$ is positive.

We consider the parameter $k^{2}$ for $\left(z_{1}, w\right) \in[-1,1] \times[-1+\delta, 1-\delta]$ with certain small $\delta$. The values $w=\frac{a}{b}=\mp 1$ correspond (as Fig. 2 shows) to the upright and inverted spinning solutions, which are asymptotic solutions of TT equations and are never attained during the inversion.

The inverting TT starts with $w_{0}=-1+\delta_{0}$ and angle $\theta_{0}$ close to 0 and moves to the value $w_{1}=1-\delta_{1}$ where the angle $\theta_{1}$ is close to $\pi$. So we need to estimate the function $h_{1}\left(z_{1}, w\right)=-\frac{\left(1+z_{1} w\right)\left(w+z_{1}\right)\left(1-z_{1}^{2}\right)}{\left(1+w^{2}+2 w z_{1}\right)^{2}}$, being a factor in front of $\epsilon$ in $(31)$, on a rectangle $[-1,1] \times$ $[-1+\delta, 1-\delta]=: R_{\delta}$ with $\delta<\min \left\{\delta_{0}, \delta_{1}\right\}$. The function $h_{1}\left(z_{1}, w\right)$ has on $[-1,1] \times(-1,1)$ two critical points $\left(\frac{1}{\sqrt{3}}, 0\right),\left(-\frac{1}{\sqrt{3}}, 0\right)$, which gives a local minimum $h_{1}\left(\frac{1}{\sqrt{3}}, 0\right)=-\frac{2}{3 \sqrt{3}}$ and a local maximum $h_{1}\left(-\frac{1}{\sqrt{3}}, 0\right)=\frac{2}{3 \sqrt{3}}$. In the whole region $R_{\delta}$ the function $h\left(z_{1}, w\right)$ satisfies the inequality $-\frac{2}{3 \sqrt{3}} \leq h_{1}\left(z_{1}, w\right) \leq \frac{2}{3 \sqrt{3}}$ and, therefore, it is bounded $\left|h_{1}\left(z_{1}, w\right)\right| \leq \frac{2}{3 \sqrt{3}}$. This clarifies how $k^{2}$ depends on the small parameter $\epsilon$.

To estimate $\frac{1}{\sqrt{z_{2}-z_{3}}}$ in $(28)$, we write

$$
\begin{aligned}
\frac{1}{\sqrt{z_{2}-z_{3}}}= & \frac{1}{\sqrt{2}}\left(\frac{\left(a^{2}+b^{2}+2 a b z_{1}\right)^{2}}{4 \beta^{2}\left(1-z_{1}^{2}\right)^{2}}+1-\frac{\left(\left(a^{2}+b^{2}\right) z_{1}+2 a b\right)}{\beta\left(1-z_{1}^{2}\right)}\right)^{-1 / 4}=\frac{\sqrt{\beta\left(1-z_{1}^{2}\right)}}{b \sqrt{1+w^{2}+2 w z_{1}}} \\
& \times\left(1+\frac{\left(1-z_{1}^{2}\right)^{2}}{\left(1+w^{2}+2 w z_{1}\right)^{2}} \epsilon^{2}-\frac{2\left(1-z_{1}^{2}\right)\left(\left(1+w^{2}\right) z_{1}+2 w\right)}{\left(1+w^{2}+2 w z_{1}\right)^{2}} \epsilon\right)^{-1 / 4} \\
= & \sqrt{\frac{\epsilon}{2}} \frac{\sqrt{\left(1-z_{1}^{2}\right)}}{\sqrt{1+w^{2}+2 w z_{1}}}\left(1+\frac{\left(1-z_{1}^{2}\right)\left(\left(1+w^{2}\right) z_{1}+2 w\right)}{2\left(1+w^{2}+2 w z_{1}\right)^{2}} \epsilon+O\left(\epsilon^{2}\right)\right) .
\end{aligned}
$$

The function $h_{2}\left(z_{1}, w\right)=\frac{\left(1-z_{1}^{2}\right)\left(\left(1+w^{2}\right) z_{1}+2 w\right)}{2\left(1+w^{2}+2 w z_{1}\right)^{2}}$ satifies the inequalities $-1<h_{2}\left(z_{1}, w\right)<1$ on the rectangle $\left(z_{1}, w\right) \in R_{\delta}$, so it is bounded: $\left|h_{2}\left(z_{1}, w\right)\right| \leq 1$.

Thus the nutational period behaves as

$$
\begin{aligned}
T(\epsilon)= & \frac{4 \sqrt{g\left(z^{*}\right)}}{\sqrt{m g R \alpha}} \frac{1}{\sqrt{z_{2}-z_{3}}} \int_{0}^{1} \frac{d s}{\sqrt{\left(1-s^{2}\right)\left(1-k^{2} s^{2}\right)}} \\
= & \frac{4 \sqrt{g\left(z^{*}\right)}}{\sqrt{m g R \alpha}} \sqrt{\frac{\epsilon}{2}} \frac{\sqrt{1-z_{1}^{2}}}{\sqrt{1+w^{2}+2 w z_{1}}}\left[1+h_{2}\left(z_{1}, w\right) \epsilon+O\left(\epsilon^{2}\right)\right] \\
& \times \frac{\pi}{2}\left[1+\frac{1}{4} h_{1}\left(z_{1}, w\right) \epsilon+O\left(\epsilon^{2}\right)\right] \\
= & \frac{2 \pi \sqrt{g\left(z^{*}\right)}}{\sqrt{2 m g R \alpha}} \sqrt{\frac{1-z_{1}^{2}}{1+w^{2}+2 w z_{1}}} \sqrt{\epsilon}[1+O(\epsilon)],
\end{aligned}
$$

where $z^{*}$ is some value between $z_{1}$ and $z_{2}$. In the leading factor at $\sqrt{\epsilon}$ we have $\left|\frac{1-z_{1}^{2}}{1+w^{2}+2 w z_{1}}\right| \leq 1$ on any rectangle $R_{\delta}$ and the function $g\left(z^{*}\right)$ is decreasing for $z^{*} \in(-1,1)$ with supremum $g(-1)=\frac{I_{3}(\alpha+1-\gamma)^{2}}{2\left(\gamma+\alpha^{2}-1\right)}$ so that

$$
\frac{2 \pi \sqrt{g\left(z^{*}\right)}}{\sqrt{2 m g R \alpha}} \sqrt{\frac{1-z_{1}^{2}}{1+w^{2}+2 w z_{1}}} \sqrt{\epsilon}<\frac{2 \pi \sqrt{g(-1)} \sqrt{\epsilon}}{\sqrt{2 m g R \alpha}}=2 \pi\left(\frac{R I_{3} \gamma(\alpha+1-\gamma)}{b}\right)=: T_{\max } .
$$

We summarize these results in a proposition. 


\section{Proposition 3.}

i) For $D \in\left(D_{1}, D_{0}\right)=\left(-\frac{\lambda \sqrt{d(-1)}}{R(1+\alpha)}, \frac{\lambda \sqrt{d(1)}}{R(1-\alpha)}\right), \tilde{E}>V\left(z_{\min }, D, \lambda\right)$ with

$$
\lambda=C \lambda_{\text {thres }}=C \frac{\sqrt{m g R^{3} I_{3} \alpha}(1+\alpha)^{2}}{\sqrt{1+\alpha-\gamma}}, \quad C>1,
$$

the period of oscillations behaves as

$$
\frac{2 \pi \sqrt{g\left(z^{*}\right)}}{\sqrt{2 m g R \alpha}} \sqrt{\frac{1-z_{1}^{2}}{1+w^{2}+2 w z_{1}}} \sqrt{\epsilon}[1+O(\epsilon)], \quad \text { as } \quad \epsilon=\frac{2 \beta}{b^{2}} \rightarrow 0 .
$$

ii) The leading factor is bounded

$$
\frac{2 \pi \sqrt{g\left(z^{*}\right)}}{\sqrt{2 m g R \alpha}} \sqrt{\frac{1-z_{1}^{2}}{1+w^{2}+2 w z_{1}}} \sqrt{\epsilon} \leq \frac{2 \pi R I_{3} \gamma(\alpha+1-\gamma)}{b}=: T_{\max }
$$

for $w$ and $z_{1}$ in any closed rectangle $\left(z_{1}, w\right) \in[-1,1] \times[-1+\delta, 1-\delta]$.

Remark 1. Notice that the upper bound is taken uniformly w.r.t. both variables $\left(z_{1}, w\right)$ over $R_{\delta}$. It does not take into account the dependence of the root $z_{1}(a, b)$ on $a, b$ and that $z^{*} \in\left[z_{1}, z_{2}\right]$ belongs to a subinterval of $[-1,1]$.

A better estimate could be difficult to find due to the complexity of the expression for $V(z, D, \lambda)$. It is actually not needed when we do qualitative analysis of oscillations within a deforming potential. Here we wanted to see how the period of oscillations within the potential $V(z, D, \lambda)$ depends on the value of Jellett's integral $\lambda$, as stated in Proposition 3 , in order to relate the time of inversion $T_{\mathrm{inv}}$ to this period. The dependence $T_{\max } \sim \frac{1}{b} \sim \frac{1}{\lambda}$ implies that the frequency of oscillations within the potential behaves as $\frac{2 \pi}{T_{\max }} \sim \lambda$.

For formulating a sufficient condition for having oscillating behaviour of $\theta(t)$ we need to know that there is an upper bound for the period of oscillations within $V(z, D, \lambda)$. To find a universal bound independent of choice of $\lambda>\lambda_{\text {thres }}$ could be difficult because the functions $h_{1}$ and $h_{2}$ have singularities at the boundary of the rectangle $\left(z_{1}, w\right) \in[-1,1] \times[-1,1]$. Finding a universal bound would require detailed analysis of the interdependence between $z_{1}$ and $w$ during inversion. Therefore we restrict our estimate to the region $w \in[-1+\delta, 1-\delta]$ with a certain suitable $\delta$ and $\epsilon<\frac{1}{C^{2}}$, meaning $\lambda>C \lambda_{\text {thres }}$ (see (24)).

We consider period $T$ given by (28) as a function of $\epsilon, w=\frac{a}{b}$ and $z_{1} \in[-1,1]$, but we drop here the assumption $\epsilon \rightarrow 0$. Let us take $\epsilon<0.9, w \in[-1+\delta, 1-\delta]$ with $\delta=0.0001$. These are physically well justified values since $\epsilon<0.9$ means $\lambda>1.054 \lambda_{\text {thres }}$ and $w= \pm 0.9999$ corresponds to extremely vertical initial angular momentum $\mathbf{L}$ that is practically never taken by a toy TT.

For $\left(z_{1}, w\right) \in R_{\delta}=[-1,1] \times[-1+\delta, 1-\delta]$ with $\delta=0.0001$ and $\epsilon<0.9$, the function appearing in the calculation of $\frac{1}{\sqrt{z_{2}-z_{3}}}(32)$ :

$$
h_{3}\left(z_{1}, w\right)=\left(1+\frac{\left(1-z_{1}^{2}\right)^{2}}{\left(1+w^{2}+2 w z_{1}\right)^{2}} \epsilon^{2}-\frac{2\left(1-z_{1}^{2}\right)\left(\left(1+w^{2}\right) z_{1}+2 w\right)}{\left(1+w^{2}+2 w z_{1}\right)^{2}} \epsilon\right)^{-1 / 4},
$$

can be shown, by checking the maximum and minimum using a computer algebra system since $h_{3}$ does not have singularities in $R_{\delta}$, to satisfy $0<h_{3}\left(z_{1}, w\right)<3.15$. Then we get

$$
\frac{1}{\sqrt{z_{2}-z_{3}}}=\sqrt{\epsilon} \frac{1}{\sqrt{2}} \sqrt{\frac{1-z_{1}^{2}}{1+w^{2}+2 w z_{1}}} h_{3}\left(z_{1}, w\right) \leq 2.23 \sqrt{\epsilon}
$$


The parameter $k^{2}$ (30) can similarly be shown to satisfy $0<k^{2}<0.342$ in this $R_{\delta}$, which implies that the elliptic integral in $(29)$ satisfies $\frac{\pi}{2}<K\left(k^{2}\right)<1.74$. Thus for the period $T(\epsilon)$ we have the estimate

$$
T(\epsilon) \leq 2.23 \cdot 1.74 \frac{4 \sqrt{g\left(z^{*}\right)}}{\sqrt{m g R \alpha}} \sqrt{\epsilon}<21.95\left(\frac{R I_{3} \gamma(\alpha+1-\gamma)}{b}\right)=: T_{\mathrm{upp}},
$$

provided that $\epsilon<0.9$.

Notice that $T_{\text {upp }}=\frac{21.95}{2 \pi} T_{\max }$ is (as expected) larger than $T_{\max }$ that is providing a bound for the leading factor of $T(\epsilon)$.

Proposition 4. Let $\left(z_{1}, w\right) \in R_{\delta}$ with $\delta=0.0001, \epsilon<0.9$ so that $b^{2}>\frac{20 \beta}{9}$, which corresponds to $\lambda>1.054 \lambda_{\text {thres }}$. Then the period of oscillations is bounded by $T(\epsilon)<T_{\mathrm{upp}}=$ $21.95\left(\frac{R I_{3} \gamma(\alpha+1-\gamma)}{b}\right)$.

The estimate $T_{\text {upp }}$ is not the best possible estimate because it is taken uniformly over $R_{\delta}$, but it is of the same order of magnitude as $T_{\max }\left(T_{\text {upp }} \approx 3.5 T_{\max }\right)$ that also has been taken uniformly. For large angular velocities (implying small $\epsilon$ ) $T_{\max }$ provides better estimate of magnitude of period of oscillation. Real oscillations within $V(z, D, \lambda)$, as observed in numerical simulations, for most of the time have shorter period.

If the time of inversion is an order of magnitude larger than $T_{\mathrm{upp}}$, say $T_{\mathrm{inv}}>10 T_{\mathrm{upp}}$, then $\theta(t)$ changes sign many times and it has oscillatory behaviour.

It should be stressed that behaviour of a correctly built TT with $1-\alpha<\gamma<1+\alpha$ depends strongly on the initial conditions and it also depends on the friction function $\mu\left(\mathbf{s}, \mathbf{L}, \hat{\mathbf{3}}, \mathbf{v}_{A}, t\right)$. The majority of solutions do not demonstrate inverting behaviour and even inverting solutions do not necessarily have to be oscillatory. The estimate found here provide a natural condition for the oscillatory behaviour of TT.

Notice the dependence of $T_{\max } \sim \frac{1}{\lambda}$ and $T_{\text {upp }} \sim \frac{1}{\lambda}$. A high initial angular momentum $\mathbf{L}$ with $\mathbf{L}$ almost parallel to the vertical axis $\hat{z}$ implies a high value of Jellett's integral $\lambda=$ $R I_{1} \dot{\varphi} \sin ^{2} \theta-R I_{3} \omega_{3}(\alpha-\cos \theta)$ since $-R I_{3} \omega_{3}(\alpha-\cos \theta)>0$. Then the period of nutations becomes small and $\theta(t)$ performs many oscillations within a given inversion time $T_{\text {inv }}$.

Example 2. By using again parameters from Example 1, we see that $T_{\max }=\frac{2 \pi R I_{3} \gamma(\alpha+1-\gamma)}{b}=$ $2.1816 \cdot 10^{-8} \cdot \frac{2 \pi}{b}$. Since $b$ has the lower bound $\frac{\lambda \gamma \alpha}{1+\alpha}=1.4851 \cdot 10^{-6}$ and the upper bound $\frac{\lambda \gamma \alpha}{1-\alpha}=2.7581 \cdot 10^{-6}$, the maximal period of nutation satisfies $0.0497<T_{\max }<0.0923$ seconds.

\section{Conclusions}

An analysis of the dynamics of an inverting top has been performed in the special case $1-\alpha<$ $1-\alpha^{2}<\gamma<1<1+\alpha$ and $I_{1}=\frac{I_{3}^{2}+m R^{2} I_{3}\left(1-\alpha^{2}\right)}{I_{3}+m R^{2}}$ for which the effective potential $V(z, D(t), \lambda)$ is a rational function of $z=\cos \theta$.

We have shown that this potential is strictly convex, has only one minimum (Proposition 1) and that this minimum $z_{\min }$ moves from the interval $[1-\epsilon, 1]$ to the interval $[-1,-1+\epsilon]$ (Proposition 2).

The high frequency period of oscillations $T_{\max }$ and the maximal period of oscillations $T_{\mathrm{upp}}$ within the potential $V(z, D, \lambda)$ has been estimated in Propositions 3,4 .

When the time of inversion $T_{\mathrm{inv}}$ is an order of magnitude larger than the maximal period of oscillations $T_{\max }$ within the potential $V(z, D, \lambda)$, then the velocity $\dot{\theta}(t)$ has to change sign many times and the solution is oscillatory. It is visible as nutational motion of the symmetry axis $\hat{\mathbf{3}}$ on the unit sphere $S^{2}$ between two latitudes while the nutational band moves from a neighborhood of the north pole into the neighborhood of the south pole when TT inverts. 


\section{Acknowledgements}

We would like to thank Hans Lundmark for discussions and for valuable help with numerical simulations.

\section{References}

[1] Bou-Rabee N.M., Marsden J.E., Romero L.A., Tippe top inversion as a dissipation-induced instability, SIAM J. Appl. Dyn. Syst. 3 (2004), 352-377.

[2] Chaplygin S.A., On a motion of a heavy body of revolution on a horizontal plane, Regul. Chaotic Dyn. 7 (2002), 119-130.

[3] Cohen R.J., The tippe top revisited, Amer. J. Phys. 45 (1977), 12-17.

[4] Del Campo A.R., Tippe top (topsy-turnee top) continued, Amer. J. Phys. 23 (1955), 544-545.

[5] Ebenfeld S., Scheck F., A new analysis of the tippe top: asymptotic states and Liapunov stability, Ann. Physics 243 (1995), 195-217, chao-dyn/9501008.

[6] Erdélyi A., Magnus W., Oberhettinger F., Tricomi F.G., Higher transcendental functions, Vol. II, McGrawHill, New York - Toronto - London, 1953.

[7] Glad S.T., Petersson D., Rauch-Wojciechowski S., Phase space of rolling solutions of the tippe top, SIGMA 3 (2007), 041, 14 pages, nlin.SI/0703016.

[8] Jones E., Oliphant T., Peterson P., Open source scientific tools for Python, available at http://www.scipy . org.

[9] Karapetyan A.V., Qualitative investigation of the dynamics of a top on a plane with friction, J. Appl. Math. Mech. 55 (1991), 563-565.

[10] Karapetyan A.V., Rubanovskii V.N., On the stability of stationary motions of non-conservative mechanical systems, J. Appl. Math. Mech. 50 (1986), 30-35.

[11] Or A.C., The dynamics of a tippe top, SIAM J. Appl. Math. 54 (1994), 597-609.

[12] Rauch-Wojciechowski S., What does it mean to explain the rising of the tippe top?, Regul. Chaotic Dyn. 13 (2008), 316-331.

[13] Rauch-Wojciechowski S., Sköldstam M., Glad T., Mathematical analysis of the tippe top, Regul. Chaotic Dyn. 10 (2005), 333-362.

[14] Rutstam N., Study of equations for tippe top and related rigid bodies, Linköping Studies in Science and Technology, Theses No. 1106, Matematiska Institutionen, Linköpings Universitet, 2010, available at http: //swepub.kb.se/bib/swepub:oai:DiVA.org:liu-60835.

[15] Rutstam N., Tippe top equations and equations for the related mechanical systems, SIGMA 8 (2012), 019, 22 pages, arXiv:1204.1123.

[16] Rutstam N., High frequency behavior of a rolling ball and simplification of the separation equation, Regul. Chaotic Dyn. 18 (2013), 226-236.

[17] Sturm C., Mémoire sur la résolution des équations numériques, Bull. de Ferussac 11 (1829), 419-425.

[18] Ueda T., Sasaki K., Watanabe S., Motion of the tippe top: gyroscopic balance condition and stability, SIAM J. Appl. Dyn. Syst. 4 (2005), 1159-1194, physics/0507198. 